\title{
Eigenvalues and eigenvectors for a $G$-frame operator
}

\author{
Azam Yousefzadeheyni(iD, Mohammad Reza Abdollahpour* (D) \\ Department of Mathematics, Faculty of Sciences, University of Mohaghegh Ardabili, Ardabil 56199-11367, \\ Iran
}

\begin{abstract}
In this paper, we investigate eigenvalues and eigenvectors of the $g$-frame operator of $\left\{\Lambda_{j} P \in B\left(K, H_{j}\right): j \in \mathbb{J}\right\}$, where $\left\{\Lambda_{j} \in B\left(H, H_{j}\right): j \in \mathbb{J}\right\}$ is a $g$-frame for an $N$ dimensional Hilbert space $H$ and $P$ is a rank $k$ orthogonal projection of $H$ onto $K$, a closed subspace of $H$.
\end{abstract}

Mathematics Subject Classification (2010). 42C99, 47A20

Keywords. eigenvalue, eigenvector, $g$-frame, $g$-frame operator, tight $g$-frame

\section{Introduction}

Frames for Hilbert spaces were introduced by Duffin and Schaeffer [2] in 1952. They used frames as a tool in the study of nonharmonic Fourier analysis. In 2006, $g$-frame as a generalization of frame was introduced and investigated by Sun [5]. For more references on $g$-frames, we can refer to $[3,6,7]$. The authors of [1] discussed eigenvectors of a frame operator in a finite dimensional Hilbert space. In this paper, we study eigenvalues and eigenvectors of a $g$-frame operator in a finite dimensional Hilbert space.

Throughout this paper, $H$ is an $N$-dimensional Hilbert space and $\left\{H_{j}\right\}_{j \in \mathbb{J}}$ is a finite sequence of Hilbert spaces, where $\mathbb{J}$ is a finite subset of $\mathbb{N}$. We denote the space of all bounded linear operators from $H$ into $H_{j}$ by $B\left(H, H_{j}\right)$.

Definition 1.1 ([5]). A sequence of operators $\Lambda=\left\{\Lambda_{j} \in B\left(H, H_{j}\right): j \in \mathbb{J}\right\}$ is called a $g$-frame for $H$ with respect to $\left\{H_{j}\right\}_{j \in \mathbb{J}}$, if there exist two constants $0<A \leq B<\infty$, such that

$$
A\|f\|^{2} \leq \sum_{j \in \mathbb{J}}\left\|\Lambda_{j} f\right\|^{2} \leq B\|f\|^{2}, \quad f \in H,
$$

$A$ and $B$ are called the lower and upper $g$-frame bounds, respectively.

We call $\Lambda=\left\{\Lambda_{j} \in B\left(H, H_{j}\right): j \in \mathbb{J}\right\}$ a tight $g$-frame if $A=B$ and a Parseval $g$-frame if

\footnotetext{
* Corresponding Author.

Email addresses: azamyousefzadeh@gmail.com (A. Yousefzadeheyni), mrabdollahpour@yahoo.com (M.R. Abdollahpour)

Received: 18.07.2018; Accepted: 10.09.2019
} 
$A=B=1$. If the right hand inequality of (1.1) holds for all $f \in H$ then we say that $\Lambda$ is a $g$-Bessel sequence. Let us consider the space

$$
\widehat{H}=\left\{\left\{f_{j}\right\}_{j \in \mathbb{J}}: f_{j} \in H_{j}, j \in \mathbb{J}, \sum_{j \in \mathbb{J}}\left\|f_{j}\right\|^{2}<+\infty\right\}
$$

with the inner product given by $\left\langle\left\{f_{j}\right\}_{j \in \mathbb{J}},\left\{g_{j}\right\}_{j \in \mathbb{J}}\right\rangle=\sum_{j \in \mathbb{J}}\left\langle f_{j}, g_{j}\right\rangle$. It is easy to show that $\widehat{H}$ is a Hilbert space with respect to the pointwise operations. It is proved in [4], if $\Lambda=\left\{\Lambda_{j} \in B\left(H, H_{j}\right): j \in \mathbb{J}\right\}$ is a $g$-Bessel sequence for $H$ then the operator

$$
T_{\Lambda}: \widehat{H} \longrightarrow H, \quad T_{\Lambda}\left(\left\{f_{j}\right\}_{j \in \mathbb{J}}\right)=\sum_{j \in \mathbb{J}} \Lambda_{j}^{*}\left(f_{j}\right),
$$

is well defined and bounded and its adjoint is

$$
T_{\Lambda}^{*}: H \longrightarrow \widehat{H}, \quad T_{\Lambda}^{*} f=\left\{\Lambda_{j} f\right\}_{j \in \mathbb{J}} .
$$

Also, a sequence $\Lambda=\left\{\Lambda_{j} \in B\left(H, H_{j}\right): j \in \mathbb{J}\right\}$ is a $g$-frame for $H$ if and only if the operator $T_{\Lambda}$ defined by (1.2) is bounded and onto. We call the operators $T_{\Lambda}$ and $T_{\Lambda}^{*}$, the synthesis and analysis operators of $\Lambda$, respectively. If $\Lambda=\left\{\Lambda_{j} \in B\left(H, H_{j}\right): j \in \mathbb{J}\right\}$ is a $g$-frame for $H$, then

$$
S_{\Lambda}: H \longrightarrow H, \quad S_{\Lambda} f=\sum_{j \in \mathbb{J}} \Lambda_{j}^{*} \Lambda_{j} f
$$

is a bounded invertible positive operator [5]. $S_{\Lambda}$ is called the $g$-frame operator of $\Lambda$ and

$$
f=\sum_{j \in \mathbb{J}} S_{\Lambda}^{-1} \Lambda_{j}^{*} \Lambda_{j} f=\sum_{j \in \mathbb{J}} \Lambda_{j}^{*} \Lambda_{j} S_{\Lambda}^{-1} f, \quad f \in H .
$$

Here we state the following lemma and omit its proof.

Lemma 1.2. If $\left\{\Lambda_{j} \in B\left(H, H_{j}\right): j \in \mathbb{J}\right\}$ is a g-frame for $H$ and $P$ is an orthogonal projection of $H$ onto $K$, a closed subspace of $H$, then $\left\{\Lambda_{j} P \in B\left(K, H_{j}\right): j \in \mathbb{J}\right\}$ is a $g$-frame for $K$.

\section{Main results}

In this section, we give some classifications of eigenvalues and eigenvectors of the $g$ frame operator of $\left\{\Lambda_{j} P \in B\left(H, H_{j}\right): j \in \mathbb{J}\right\}$ where $\left\{\Lambda_{j} \in B\left(H, H_{j}\right): j \in \mathbb{J}\right\}$ is a $g$-frame for $H$ and $P$ is an orthogonal projection on $H$.

Proposition 2.1. Let $\left\{\Lambda_{j} \in B\left(H, H_{j}\right): j \in \mathbb{J}\right\}$ be a g-frame for $H$. Let $e_{1} \in H,\left\|e_{1}\right\|=1$ and let $P$ be the orthogonal projection of $H$ onto span $\left\{e_{1}\right\}$. Then the following statements are equivalent:

(1) $e_{1}$ is an eigenvector for $S_{\Lambda}$ with the eigenvalue $\lambda_{1}$.

(2) $\sum_{j \in \mathbb{J}}\left\|\Lambda_{j} e_{1}\right\|^{2}=\lambda_{1}$ and $\sum_{j \in \mathbb{J}}\left\langle\Lambda_{j} e_{1}, \Lambda_{j} f\right\rangle=0$ for all $f \in(I-P) H$.

Proof. Assume that (1) holds; we have

$$
\sum_{j \in \mathbb{J}}\left\|\Lambda_{j} e_{1}\right\|^{2}=\left\langle\sum_{j \in \mathbb{J}} \Lambda_{j}^{*} \Lambda_{j} e_{1}, e_{1}\right\rangle=\left\langle S_{\Lambda} e_{1}, e_{1}\right\rangle=\lambda_{1} .
$$

If $f \in(I-P) H$, then

$$
\sum_{j \in \mathbb{J}}\left\langle\Lambda_{j} e_{1}, \Lambda_{j} f\right\rangle=\left\langle S_{\Lambda} e_{1}, f\right\rangle=\lambda_{1}\left\langle e_{1}, f\right\rangle=0 .
$$


Conversely, let (2) hold; choose $\left\{e_{i}\right\}_{i=2}^{N}$ so that $\left\{e_{i}\right\}_{i=1}^{N}$ is an orthonormal basis for $H$. Then

$$
\begin{aligned}
S_{\Lambda} e_{1} & =\sum_{i=1}^{N}\left\langle S_{\Lambda} e_{1}, e_{i}\right\rangle e_{i}=\sum_{i=1}^{N} \sum_{j \in \mathbb{J}}\left\langle\Lambda_{j} e_{1}, \Lambda_{j} e_{i}\right\rangle e_{i} \\
& =\sum_{j \in \mathbb{J}}\left(\left\|\Lambda_{j} e_{1}\right\|^{2}\right) e_{1}+\sum_{i=2}^{N}\left(\sum_{j \in \mathbb{J}}\left\langle\Lambda_{j} e_{1}, \Lambda_{j} e_{i}\right\rangle\right) e_{i}=\lambda_{1} e_{1} .
\end{aligned}
$$

Therefore $e_{1}$ is an eigenvector for $S_{\Lambda}$ with the eigenvalue $\lambda_{1}$.

Proposition 2.2. Let $\left\{\Lambda_{j} \in B\left(H, H_{j}\right): j \in \mathbb{J}\right\}$ be a g-frame for $H$. Let $\left\{e_{i}\right\}_{i=1}^{N}$ be an orthonormal basis for $H$ and $e_{i}$ be an eigenvector of $S_{\Lambda}$ with the eigenvalue $\lambda_{i}$, for $i=1,2, \ldots, N$. Then

$$
\sum_{j \in \mathbb{J}}\left\|\Lambda_{j} f\right\|^{2}=\sum_{i=1}^{N} \lambda_{i}\left|\left\langle f, e_{i}\right\rangle\right|^{2}, \quad f \in H
$$

and

$$
\sum_{j \in \mathbb{J}}\left\langle\Lambda_{j} f, \Lambda_{j} g\right\rangle=\sum_{i=1}^{N} \lambda_{i}\left\langle f, e_{i}\right\rangle \overline{\left\langle g, e_{i}\right\rangle}, \quad f, g \in H .
$$

Proof. We have

$$
\begin{aligned}
\sum_{j \in \mathbb{J}}\left\|\Lambda_{j} f\right\|^{2} & =\left\langle S_{\Lambda} f, f\right\rangle \\
& =\left\langle S_{\Lambda}\left(\sum_{i=1}^{N}\left\langle f, e_{i}\right\rangle e_{i}\right), \sum_{j=1}^{N}\left\langle f, e_{j}\right\rangle e_{j}\right\rangle \\
& =\sum_{i=1}^{N} \sum_{j=1}^{N} \lambda_{i}\left\langle f, e_{i}\right\rangle \overline{\left\langle f, e_{j}\right\rangle}\left\langle e_{i}, e_{j}\right\rangle \\
& =\sum_{i=1}^{N} \lambda_{i}\left|\left\langle f, e_{i}\right\rangle\right|^{2},
\end{aligned}
$$

for all $f \in H$. On the other hand

$$
\begin{aligned}
\sum_{j \in \mathbb{J}}\left\langle\Lambda_{j} f, \Lambda_{j} g\right\rangle & =\left\langle S_{\Lambda} f, g\right\rangle \\
& =\left\langle S_{\Lambda}\left(\sum_{i=1}^{N}\left\langle f, e_{i}\right\rangle e_{i}\right), \sum_{j=1}^{N}\left\langle g, e_{j}\right\rangle e_{j}\right\rangle \\
& =\sum_{i=1}^{N} \sum_{j=1}^{N} \lambda_{i}\left\langle f, e_{i}\right\rangle \overline{\left\langle g, e_{j}\right\rangle}\left\langle e_{i}, e_{j}\right\rangle \\
& =\sum_{i=1}^{N} \lambda_{i}\left\langle f, e_{i}\right\rangle \overline{\left\langle g, e_{i}\right\rangle},
\end{aligned}
$$

for all $f, g \in H$.

Theorem 2.3. Let $\left\{\Lambda_{j} \in B\left(H, H_{j}\right): j \in \mathbb{J}\right\}$ be a g-frame for $H$. Fix $1 \leq k \leq N$ and let $P$ be a rank $k$ orthogonal projection of $H$ onto a subspace $K$. Let $S_{1}$ be the $g$-frame operator of $\left\{\Lambda_{j} P \in B\left(K, H_{j}\right): j \in \mathbb{J}\right\}$ and choose orthonormal family $\left\{f_{i}\right\}_{i=1}^{k}$ in $K$. Then the following statements are equivalent: 
(1) $\left\{f_{i}\right\}_{i=1}^{k}$ is a family of eigenvectors of $S_{1}$ with eigenvalues $\left\{\mu_{i}\right\}_{i=1}^{k}$.

(2) We have

(i) $\sum_{j \in \mathbb{J}}\left\|\Lambda_{j} f_{i}\right\|^{2}=\mu_{i}, \quad 1 \leq i \leq k$,

(ii) $\sum_{j \in \mathbb{J}}\left\langle\Lambda_{j} f_{i}, \Lambda_{j} f_{l}\right\rangle=0, \quad 1 \leq i \neq l \leq k$.

Proof. Let (1) hold. Then

$$
\sum_{j \in \mathbb{J}}\left\|\Lambda_{j} f_{i}\right\|^{2}=\sum_{j \in \mathbb{J}}\left\|\Lambda_{j} P f_{i}\right\|^{2}=\left\langle S_{1} f_{i}, f_{i}\right\rangle=\mu_{i}\left\|f_{i}\right\|^{2}=\mu_{i},
$$

for $i=1,2, \ldots, k$. Similarly,

$$
\sum_{j \in \mathbb{J}}\left\langle\Lambda_{j} f_{i}, \Lambda_{j} f_{l}\right\rangle=\left\langle S_{1} f_{i}, f_{l}\right\rangle=\mu_{i}\left\langle f_{i}, f_{l}\right\rangle=0, \quad 1 \leq i \neq l \leq k .
$$

Now, assume that (2) is true. Then choose $\left\{f_{i}\right\}_{i=k+1}^{N}$ so that $\left\{f_{i}\right\}_{i=1}^{N}$ is an orthonormal basis for $H$. Then

$$
\begin{aligned}
S_{1} f_{i} & =\sum_{l=1}^{N}\left\langle S_{1} f_{i}, f_{l}\right\rangle f_{l}=\sum_{l=1}^{N}\left\langle\sum_{j \in \mathbb{J}} P \Lambda_{j}^{*} \Lambda_{j} P f_{i}, f_{l}\right\rangle f_{l} \\
& =\sum_{l=1}^{k} \sum_{j \in \mathbb{J}}\left\langle\Lambda_{j} f_{i}, \Lambda_{j} f_{l}\right\rangle f_{l} \\
& =\left(\sum_{j \in \mathbb{J}}\left\langle\Lambda_{j} f_{i}, \Lambda_{j} f_{i}\right\rangle\right) f_{i}+\sum_{\substack{l=1 \\
l \neq i}}^{k}\left(\sum_{j \in \mathbb{J}}\left\langle\Lambda_{j} f_{i}, \Lambda_{j} f_{l}\right\rangle\right) f_{l} \\
& =\left(\sum_{j \in \mathbb{J}}\left\|\Lambda_{j} f_{i}\right\|^{2}\right) f_{i}+\sum_{\substack{l=1 \\
l \neq i}}^{k}\left(\sum_{j \in \mathbb{J}}\left\langle\Lambda_{j} f_{i}, \Lambda_{j} f_{l}\right\rangle\right) f_{l} \\
& =\mu_{i} f_{i},
\end{aligned}
$$

so, $f_{i}$ is an eigenvector of $S_{1}$ with eigenvalue $\mu_{i}$ for $i=1,2, \ldots, k$.

Theorem 2.4. Let $\left\{\Lambda_{j} \in B\left(H, H_{j}\right): j \in \mathbb{J}\right\}$ be a g-frame for $H$. Let $\left\{e_{i}\right\}_{i=1}^{N}$ be an orthonormal basis for $H$ and $e_{i}$ be an eigenvector of $S_{\Lambda}$ with the eigenvalue $\lambda_{i}$, for $i=$ $1,2, \ldots, N$. Fix $1 \leq k \leq N$ and let $P$ be a rank $k$ orthogonal projection of $H$ onto $K$ subspace of $H$ and let $S_{1}$ be the $g$-frame operator of $\left\{\Lambda_{j} P \in B\left(K, H_{j}\right): j \in \mathbb{J}\right\}$ and choose orthonormal family $\left\{f_{i}\right\}_{i=1}^{k}$ in $K$. Then the following statements are equivalent:

(1) $\left\{f_{i}\right\}_{i=1}^{k}$ is a family of eigenvectors of $S_{1}$ with eigenvalues $\left\{\mu_{i}\right\}_{i=1}^{k}$.

(2) We have

$$
\begin{aligned}
& \text { (i) } \sum_{n=1}^{N} \lambda_{n}\left|\left\langle f_{i}, e_{n}\right\rangle\right|^{2}=\mu_{i}, \quad 1 \leq i \leq k, \\
& \text { (ii) } \sum_{n=1}^{N} \lambda_{n}\left\langle f_{i}, e_{n}\right\rangle \overline{\left\langle f_{j}, e_{n}\right\rangle}=0, \quad 1 \leq i \neq j \leq k .
\end{aligned}
$$

Proof. It is clear by Proposition 2.2 and Theorem 2.3. 
Proposition 2.5. Let $\left\{\Lambda_{j} \in B\left(H, H_{j}\right): j \in \mathbb{J}\right\}$ be a g-frame for $H$. Let $\left\{e_{i}\right\}_{i=1}^{N}$ be an orthonormal basis for $H$ and $e_{i}$ be an eigenvector of $S_{\Lambda}$ with eigenvalue $\lambda_{i}$ for $i=$ $1,2, \ldots, N$. Let $\left\{\sigma_{j}\right\}_{j=1}^{k}$ be a partition of $\{1,2, \ldots, N\}$ and for every $1 \leq j \leq k$ let $f_{j}=$ $\sum_{i \in \sigma_{j}} a_{i} e_{i}$ with $\left\|f_{j}\right\|=\sum_{i \in \sigma_{j}}\left|a_{i}\right|^{2}=1$. Let $P$ be the orthogonal projection of $H$ onto $K=\operatorname{span}\left\{f_{j}\right\}_{j=1}^{k}$ and let $S_{1}$ be the $g$-frame operator for $\left\{\Lambda_{j} P \in B\left(K, H_{j}\right): j \in \mathbb{J}\right\}$. Then $\left\{f_{j}\right\}_{j=1}^{k}$ is an orthonormal basis for $K$ and $f_{j}$ is an eigenvector of $S_{1}$ with eigenvalue $\mu_{j}=\sum_{i \in \sigma_{j}} \lambda_{i}\left|a_{i}\right|^{2}$ for $j=1,2, \ldots, k$.

Proof. We have

$$
\begin{aligned}
\sum_{n \in \mathbb{J}}\left\|\Lambda_{n} f_{i}\right\|^{2} & =\sum_{n \in \mathbb{J}}\left\langle\Lambda_{n} f_{i}, \Lambda_{n} f_{i}\right\rangle \\
& =\sum_{n \in \mathbb{J}}\left\langle\Lambda_{n}\left(\sum_{j \in \sigma_{i}} a_{j} e_{j}\right), \Lambda_{n}\left(\sum_{l \in \sigma_{i}} a_{l} e_{l}\right)\right\rangle \\
& =\sum_{n \in \mathbb{J}} \sum_{j \in \sigma_{i}} \sum_{l \in \sigma_{i}} a_{j} \overline{a_{l}}\left\langle\Lambda_{n} e_{j}, \Lambda_{n} e_{l}\right\rangle \\
& =\sum_{n \in \mathbb{J}} \sum_{j \in \sigma_{i}}\left|a_{j}\right|^{2}\left\langle\Lambda_{n} e_{j}, \Lambda_{n} e_{j}\right\rangle+\sum_{n \in \mathbb{J}} \sum_{\substack{j, l \in \sigma_{i} \\
j \neq l}} a_{j} \overline{a_{l}}\left\langle\Lambda_{n} e_{j}, \Lambda_{n} e_{l}\right\rangle \\
& =\sum_{j \in \sigma_{i}}\left|a_{j}\right|^{2}\left\langle S_{\Lambda} e_{j}, e_{j}\right\rangle+\sum_{\substack{j, l \in \sigma_{i} \\
j \neq l}} a_{j} \overline{a_{l}}\left\langle S_{\Lambda} e_{j}, e_{l}\right\rangle \\
& =\sum_{j \in \sigma_{i}} \lambda_{j}\left|a_{j}\right|^{2}+0=\mu_{i},
\end{aligned}
$$

for $1 \leq i \leq k$. On the other hand

$$
\begin{aligned}
\sum_{n \in \mathbb{J}}\left\langle\Lambda_{n} f_{i}, \Lambda_{n} f_{l}\right\rangle & =\sum_{n \in \mathbb{J}}\left\langle\Lambda_{n}\left(\sum_{m \in \sigma_{i}} a_{m} e_{m}\right), \Lambda_{n}\left(\sum_{t \in \sigma_{l}} a_{t} e_{t}\right)\right\rangle \\
& =\sum_{m \in \sigma_{i}} \sum_{t \in \sigma_{l}} a_{m} \overline{a_{t}}\left\langle S_{\Lambda} e_{m}, e_{t}\right\rangle \\
& =\sum_{m \in \sigma_{i}} \sum_{t \in \sigma_{l}} \lambda_{m} a_{m} \overline{a_{t}}\left\langle e_{m}, e_{t}\right\rangle=0,
\end{aligned}
$$

for $1 \leq i \neq l \leq k$. Therefore $\left\{f_{j}\right\}_{j=1}^{k}$ is an orthonormal basis for $K$ and by Theorem 2.3, $f_{j}$ is an eigenvector of $S_{1}$ with eigenvalue $\mu_{j}=\sum_{i \in \sigma_{j}} \lambda_{i}\left|a_{i}\right|^{2}$ for $j=1,2, \ldots, k$.

Proposition 2.6. Let $\left\{\Lambda_{j} \in B\left(H, H_{j}\right): j \in \mathbb{J}\right\}$ be a g-frame for $H$ with $\operatorname{dim} H=2 N$. Let $\left\{e_{i}\right\}_{i=1}^{2 N}$ be an orthonormal basis for $H$ and $e_{i}$ be an eigenvector of $S_{\Lambda}$ with eigenvalue $\lambda_{i}$, for $i=1,2, \ldots, 2 N$ and $\lambda_{2 N} \leq \lambda_{2 N-1} \leq \ldots \leq \lambda_{1}$. Let $\lambda_{2 N-i+1} \leq \mu_{i} \leq \lambda_{i}$ for $i=1,2, \ldots, 2 N$. Then there exists a subspace $K \subseteq H$ with $\operatorname{dim} K=N$ such that if $P$ is the orthogonal projection of $H$ onto $K$, then $\left\{\Lambda_{j} P \in B\left(K, H_{j}\right): j \in \mathbb{J}\right\}$ is a g-frame for $K$ with the $g$-frame operator $S_{1}$ having eigenvalues $\mu_{1}, \mu_{2}, \ldots, \mu_{N}$.

Proof. For $1 \leq i \leq N$ choose $0<\varepsilon_{i}<1$ so that $\lambda_{i} \varepsilon_{i}^{2}+\lambda_{2 N-i+1}\left(1-\varepsilon_{i}^{2}\right)=\mu_{i}$ and let $f_{i}=\varepsilon_{i} e_{i}+\sqrt{1-\varepsilon_{i}^{2}} e_{2 N-i+1}$. So, $\left\{f_{i}\right\}_{i=1}^{N}$ is an orthonormal sequence in $H$. Let $K=$ 
$\operatorname{span}\left\{f_{i}\right\}_{i=1}^{N}$. We have

$$
\begin{aligned}
& \sum_{n=1}^{2 N} \lambda_{n}\left|\left\langle f_{i}, e_{n}\right\rangle\right|^{2}=\left\langle S_{\Lambda} f_{i}, f_{i}\right\rangle \\
& \quad=\left\langle S_{\Lambda}\left(\varepsilon_{i} e_{i}+\sqrt{1-\varepsilon_{i}^{2}} e_{2 N-i+1}\right), \varepsilon_{i} e_{i}+\sqrt{1-\varepsilon_{i}^{2}} e_{2 N-i+1}\right\rangle \\
& \quad=\left\langle\varepsilon_{i} \lambda_{i} e_{i}+\sqrt{1-\varepsilon_{i}^{2}} \lambda_{2 N-i+1} e_{2 N-i+1}, \varepsilon_{i} e_{i}+\sqrt{1-\varepsilon_{i}^{2}} e_{2 N-i+1}\right\rangle \\
& \quad=\lambda_{i} \varepsilon_{i}^{2}+\lambda_{2 N-i+1}\left(1-\varepsilon_{i}^{2}\right)=\mu_{i}, \quad 1 \leq i \leq N .
\end{aligned}
$$

For $i \neq j$,

$$
\begin{aligned}
& \sum_{n=1}^{2 N} \lambda_{n}\left\langle f_{i}, e_{n}\right\rangle \overline{\left\langle f_{j}, e_{n}\right\rangle}=\left\langle S_{\Lambda} f_{i}, f_{j}\right\rangle \\
& \quad=\left\langle S_{\Lambda}\left(\varepsilon_{i} e_{i}+\sqrt{1-\varepsilon_{i}^{2}} e_{2 N-i+1}\right), \varepsilon_{j} e_{j}+\sqrt{1-\varepsilon_{j}^{2}} e_{2 N-j+1}\right\rangle \\
& \quad=\left\langle\varepsilon_{i} \lambda_{i} e_{i}+\sqrt{1-\varepsilon_{i}^{2}} \lambda_{2 N-i+1} e_{2 N-i+1}, \varepsilon_{j} e_{j}+\sqrt{1-\varepsilon_{j}^{2}} e_{2 N-j+1}\right\rangle=0 .
\end{aligned}
$$

So by Theorem 2.4, $f_{i}$ is an eigenvector for $S_{1}$ with eigenvalue $\mu_{i}$ for $i=1,2, \ldots, N$.

We close the paper by giving examples in which the conditions of Proposition 2.1 and Theorem 2.3 are satisfied.

Example 2.7. Let $H=\mathbb{C}^{3}$ and we define

$$
\begin{array}{ll}
\Lambda_{1}: \mathbb{C}^{3} \longrightarrow \mathbb{C}, & \Lambda_{1}(x, y, z)=2 x-y, \\
\Lambda_{2}: \mathbb{C}^{3} \longrightarrow \mathbb{C}, & \Lambda_{2}(x, y, z)=x+2 y, \\
\Lambda_{3}: \mathbb{C}^{3} \longrightarrow \mathbb{C}, & \Lambda_{3}(x, y, z)=\sqrt{6} z i .
\end{array}
$$

Then $\left\{\Lambda_{j} \in B\left(\mathbb{C}^{3}, \mathbb{C}\right): j=1,2,3\right\}$ is a $g$-frame for $\mathbb{C}^{3}$ with the $g$-frame bounds 5,6 . Therefore, the $g$-frame operator of $\left\{\Lambda_{j} \in B\left(\mathbb{C}^{3}, \mathbb{C}\right): j=1,2,3\right\}$ is

$$
S_{\Lambda}: \mathbb{C}^{3} \longrightarrow \mathbb{C}^{3}, \quad S_{\Lambda}(x, y, z)=(5 x, 5 y, 6 z) .
$$

Then 5 is an eigenvalue of $S_{\Lambda}$ with eigenvectors $(1,0,0),(0,1,0)$ and 6 is an eigenvalue of $S_{\Lambda}$ with eigenvector $(0,0,1)$. Let $\left\{e_{i}\right\}_{i=1}^{3}$ be the standard orthonormal basis for $\mathbb{C}^{3}$. Let us consider the orthogonal projection $P$ on $\mathbb{C}^{3}$,

$$
P: \mathbb{C}^{3} \longrightarrow \operatorname{span}\{(0,0,1)\}=\operatorname{span}\left\{e_{3}\right\} .
$$

Then

$$
\sum_{j=1}^{3}\left|\Lambda_{j}\left(e_{3}\right)\right|^{2}=0+0+|\sqrt{6} i|^{2}=6,
$$

and

$$
\sum_{j=1}^{3}\left\langle\Lambda_{j}\left(e_{3}\right), \Lambda_{j}(f)\right\rangle=\left\langle S_{\Lambda}\left(e_{3}\right), f\right\rangle=\left\langle 6 e_{3}, c_{1} e_{1}+c_{2} e_{2}\right\rangle=0, \quad f \in(I-P) \mathbb{C}^{3}
$$

Now, by the above assumption we define the orthogonal projection $P$ on $\mathbb{C}^{3}$ as follows:

$$
P: \mathbb{C}^{3} \longrightarrow K=\operatorname{span}\{(1,0,0),(0,1,0)\}, \quad P(x, y, z)=(x, y, 0) .
$$

For any $(x, y, z) \in \mathbb{C}^{3}$, we have

$$
\begin{aligned}
& \left(\Lambda_{1} P\right)(x, y, z)=2 x-y \\
& \left(\Lambda_{2} P\right)(x, y, z)=x+2 y, \\
& \left(\Lambda_{3} P\right)(x, y, z)=0 .
\end{aligned}
$$


Thus the $g$-frame operator of $\left\{\Lambda_{j} P \in B\left(\mathbb{C}^{3}, \mathbb{C}\right): j=1,2,3\right\}$ is

$$
\begin{aligned}
& S_{1}: K \longrightarrow K \\
& S_{1}(x, y, z)=P S_{\Lambda} P(x, y, z)=(5 x, 5 y, 0) .
\end{aligned}
$$

Thus $(1,0,0)$ and $(0,1,0)$ are the eigenvectors of $S_{1}$ with the eigenvalue 5 . We have

$$
\sum_{j=1}^{3}\left|\Lambda_{j}\left(e_{1}\right)\right|^{2}=5
$$

and

$$
\sum_{j=1}^{3}\left|\Lambda_{j}\left(e_{2}\right)\right|^{2}=5
$$

Also,

$$
\sum_{j=1}^{3}\left\langle\Lambda_{j}\left(e_{1}\right), \Lambda_{j}\left(e_{2}\right)\right\rangle=\left\langle S_{\Lambda}\left(e_{1}\right), e_{2}\right\rangle=\langle(5,0,0),(0,1,0)\rangle=0 .
$$

Example 2.8. Let $H=\mathbb{C}^{N}$ and we define

$$
\begin{aligned}
& \Lambda_{j}: \mathbb{C}^{N} \longrightarrow \mathbb{C}, \quad \Lambda_{j}\left(x_{1}, x_{2}, \ldots, x_{N}\right)=x_{j}, \quad 1 \leq j \leq N-1 \\
& \Lambda_{N}: \mathbb{C}^{N} \longrightarrow \mathbb{C}, \quad \Lambda_{N}\left(x_{1}, x_{2}, \ldots, x_{N}\right)=2 x_{N} .
\end{aligned}
$$

Then $\left\{\Lambda_{j} \in B\left(\mathbb{C}^{N}, \mathbb{C}\right): j=1, \ldots, N\right\}$ is a $g$-frame for $\mathbb{C}^{N}$ with the $g$-frame bounds 1,4 . Therefore, the $g$-frame operator is

$$
\begin{aligned}
& S_{\Lambda}: \mathbb{C}^{N} \longrightarrow \mathbb{C}^{N} \\
& S_{\Lambda}\left(x_{1}, x_{2}, \ldots, x_{N-1}, x_{N}\right)=\left(x_{1}, x_{2}, \ldots, x_{N-1}, 4 x_{N}\right) .
\end{aligned}
$$

The eigenvalues of $S_{\Lambda}$ are 1 and 4 and the eigenvectors of 1 are $(1,0,0, \ldots, 0),(0,1,0, \ldots, 0)$, $\ldots,(0,0, \ldots, 1,0)$ and the eigenvector of 4 is $(0,0, \ldots, 0,1)$. Let $\left\{e_{i}\right\}_{i=1}^{N}$ is the standard orthonormal basis for $\mathbb{C}^{N}$ and consider the orthogonal projection $P$ on $\mathbb{C}^{N}$ as follows:

$$
P: \mathbb{C}^{N} \longrightarrow \operatorname{span}\{(0,0, \ldots, 0,1)\} .
$$

Then

$$
\sum_{j=1}^{N}\left|\Lambda_{j}\left(e_{N}\right)\right|^{2}=4
$$

and

$$
\sum_{j=1}^{N}\left\langle\Lambda_{j}\left(e_{N}\right), \Lambda_{j}(f)\right\rangle=\left\langle S_{\Lambda}\left(e_{N}\right), f\right\rangle=\left\langle 4 e_{N}, \sum_{i=1}^{N-1} c_{i} e_{i}\right\rangle=0, \quad f \in(I-P) \mathbb{C}^{N}
$$

Now, we consider the orthogonal projection $P$ on $\mathbb{C}^{N}$,

$$
\begin{aligned}
& P: \mathbb{C}^{N} \longrightarrow K=\operatorname{span}\{(1,0,0, \ldots, 0),(0,1,0, \ldots, 0), \ldots,(0,0, \ldots, 1,0)\} \\
& P\left(x_{1}, x_{2}, \ldots, x_{N-1}, x_{N}\right)=\left(x_{1}, x_{2}, \ldots, x_{N-1}, 0\right)
\end{aligned}
$$

Then the $g$-frame operator of $\left\{\Lambda_{j} P \in B\left(\mathbb{C}^{N}, \mathbb{C}\right): j=1, \ldots, N\right\}$ is

$$
\begin{aligned}
& S_{1}: K \longrightarrow K \\
& S_{1}\left(x_{1}, x_{2}, \ldots, x_{N-1}, x_{N}\right)=\left(x_{1}, x_{2}, \ldots, x_{N-1}, 0\right) .
\end{aligned}
$$


The eigenvalues of $S_{1}$ are 0 and 1 and the eigenvector of 0 is $(0,0, \ldots, 0,1)$ and the eigenvectors of 1 are $(1,0,0, \ldots, 0),(0,1,0, \ldots, 0), \ldots,(0,0, \ldots, 1,0)$. We have

$$
\sum_{j=1}^{N}\left|\Lambda_{j}\left(e_{i}\right)\right|^{2}=1, \quad 1 \leq i \leq N-1
$$

and

$$
\sum_{j=1}^{N}\left\langle\Lambda_{j}\left(e_{l}\right), \Lambda_{j}\left(e_{i}\right)\right\rangle=\left\langle S_{\Lambda}\left(e_{l}\right), e_{i}\right\rangle=\left\langle e_{l}, e_{i}\right\rangle=0, \quad 1 \leq i \neq l \leq N-1 .
$$

Acknowledgment. The authors would like to thank the referee for his/her helpful comments to improve the quality of this manuscript.

\section{References}

[1] P. G. Cassaza and M. T. Leon, Projections of frames, Proc. SPIE 5914, 591402, 277289, 2005. Doi: 10.1117/12.615278.

[2] R. J. Duffin and A. C. Schaeffer, A class of nonharmonic Fourier series, Trans. Amer. Math. Soc. 72, 341-366, 1952.

[3] X. X. Guo, New Characterizations of g-Bessel Sequences and g-Riesz Bases in Hilbert Spaces, Result Math. 68, 361-374, 2015.

[4] A. Najati, M. H. Faroughi and A. Rahimi, G-frames and stability of g-frames in Hilbert spaces, Methods Func. Anal. Topology 4, 271-286, 2008.

[5] W. Sun, G-frames and g-Riesz bases, J. Math. Anal. Appl. 322, 437-452, 2006.

[6] X. C. Xiao, G. R. Zhou and Y. C. Zhu, Weaving of K-g-frames in Hilbert spaces, Science Asia 45, 285- 291, 2019.

[7] X. C. Xiao, Y. C. Zhu and G. R. Zhou, Characterizations of (near) exact g-frames, g-Riesz bases and Besselian g-frames, Int. J. Wavelets Multiresolut. Inf. Process. 17 (5), 1950040, 2019, https://doi.org/10.1142/S0219691319500401. 